\title{
Positive Mass Theorem for Hypersurface in 5-Dimensional Lorentzian Manifolds
}

\author{
XIAO ZHANG
}

\section{Introduction.}

It is well-known that Positive Mass Theorem has a fundamental importance in Einstein's general relativity. The positive mass theorem for 5-dimensional Lorentzian manifolds is therefore interesting in the context of Kaluza-Klein theory which provides a 5-dimensional general relativity containing both Einstein's 4-dimensional theorey of gravity and Maxwell's theory of electromagnetism. This idea of Kaluza-Klein was enthusiastically received by unified-field theorists and was extended to higher dimensions to include the strong and weak forces (i.e., 11-dimensional supergravity theories and 10-dimensional superstrings). We refer to review article [OW] for higherdimensional unified theories from the general relativity side. Mathematically, the existence of $S$ pin $^{c}$ structures on orientable 4-manifolds provides a unified treatment on gravity and electromagnetism. In this paper we adapt Witten's method and the analytic arguments of Parker and Taubes to such a Spin $^{c}$ structure. This yields a Positive Mass Theorem (Theorem 1.6 below) for hypersurfaces in 5-dimensional Lorentzian manifolds.

Let $N$ be a 5 -dimensional Lorentzian manifold with Lorentzian metric $\widetilde{g}$ of signature $(-1,1,1,1,1)$, which satisfies the Einstein equations

$$
\widetilde{R}_{\alpha \beta}-\frac{\widetilde{R}}{2} \widetilde{g}_{\alpha \beta}=T_{\alpha \beta}
$$

where $\widetilde{R}_{\alpha \beta}, \widetilde{R}$ are the Ricci and scalar curvatures of $\widetilde{g}$ respectively, $T_{\alpha \beta}$ is a symmetric tensor field which is interpreted physically as the energymomentum tensor of matter.

Definition 1.1. A spacelike hypersurface $M$ of $N$ is called asymptotically flat of order $\tau$ if there is a compact set $K \subset M$ such that $M-K$ is the disjoint union of a finite number of subsets $M_{1}, \cdots, M_{k}$ - called the "ends" 
of $M$ - each diffeomorphic to the complement of a contractible compact set in $R^{4}$. Under the diffeomorphism the metric of $M_{l} \subset M$ is of the form

$$
g_{i j}=\delta_{i j}+a_{i j}
$$

in the standard coordinates $\left\{x^{i}\right\}$ on $R^{4}$, where $a_{i j}$ satisfies

$$
a_{i j}=O\left(r^{-\tau}\right), \quad \partial_{k} a_{i j}=O\left(r^{-\tau-1}\right), \quad \partial_{l} \partial_{k} a_{i j}=O\left(r^{-\tau-2}\right) .
$$

Furthermore, the second fundamental form of $M$ satisfies

$$
h_{i j}=O\left(r^{-\tau-1}\right), \quad \partial_{k} h_{i j}=O\left(r^{-\tau-2}\right) .
$$

A $U(1)$ line bundle $L$ over $M$ is called asymptotically flat of order $\tau$ if there is a trivialization of $L$ over the end and a $u(1)$-value 1-form $A$ such that on end $M_{l}$, the connection on $L$ can be written as

$$
d_{A j}=\partial_{j}+A_{j} \mathbf{i}
$$

where $A_{j}$ is real, and satisfies

$$
A_{j}=O\left(r^{-\tau-1}\right), \quad \partial_{k} A_{j}=O\left(r^{-\tau-2}\right) .
$$

We will often identify the end $M_{l} \subset M$ with the corresponding set $M_{l} \subset R^{4}$.

The curvature $F_{A}=d A$ of such a connection on $L$ may be interpreted physically as the electromagnetic field. For spacelike asymptotically flat hypersurface $M$ and asymptotically flat line bundle $L$, we can define the total energy, the total linear momentum and the total electromagnetic momentum. They are defined in each asymtotic end $M_{l}$ as limits over the sphere $S_{R, l}$ of radius $R$ in $M_{l} \subset R^{4}$.

Definition 1.2. Total energy of end $M_{l}$ is defined as

$$
E_{l}=\lim _{R \rightarrow \infty} C_{4}^{-1} \int_{S_{R, l}}\left(\partial_{j} g_{i j}-\partial_{i} g_{j j}\right) d \Omega^{i}
$$

total linear momentum of end $M_{l}$ is defined as

$$
p_{l k}=\lim _{R \rightarrow \infty} C_{4}^{-1} \int_{S_{R, l}} 2\left(h_{i k}-\delta_{i k} h_{j j}\right) d \Omega^{i},
$$

total electromagnetic momentum of end $M_{l}$ is defined as

$$
q_{l i j}=\lim _{R \rightarrow \infty} C_{4}^{-1}\left(\int_{S_{R, l}} 2 A_{j} d \Omega^{i}-\int_{S_{R, l}} 2 A_{i} d \Omega^{j}\right),
$$

where $C_{4}=12 \omega_{3}$ and $\omega_{3}$ is the volume of unit sphere $S^{3}$ with standard metric. 
Definition 1.3. The current matrix of electromagnetic field on end $M_{l}$ is defined by

$$
\Omega_{l}=\left(\omega_{l i j}\right)
$$

where

$$
\begin{aligned}
& \omega_{l 11}=2^{-1}\left(-q_{l 12}^{2}-q_{l 13}^{2}-q_{l 14}^{2}+q_{l 34}^{2}+q_{l 42}^{2}+q_{l 23}^{2}\right), \\
& \omega_{l 22}=2^{-1}\left(-q_{l 12}^{2}+q_{l 13}^{2}+q_{l 14}^{2}+q_{l 34}^{2}-q_{l 42}^{2}-q_{l 23}^{2}\right), \\
& \omega_{l 33}=2^{-1}\left(q_{l 12}^{2}-q_{l 13}^{2}+q_{l 14}^{2}-q_{l 34}^{2}+q_{l 42}^{2}-q_{l 23}^{2}\right), \\
& \omega_{l 44}=2^{-1}\left(q_{l 12}^{2}+q_{l 13}^{2}-q_{l 14}^{2}-q_{l 34}^{2}-q_{l 42}^{2}+q_{l 23}^{2}\right), \\
& \omega_{l i j}=\sum_{k \neq\{i, j\}} q_{l i k} q_{l k j}, \quad 1 \leq i, j \leq 4, i \neq j .
\end{aligned}
$$

When the asymptotic order $\tau>1$, these quantities are finite, independent on the choice of asymptotic coordinates. Since $q_{l i j}=-q_{l j i}, \Omega_{l}$ is real symmetric. Moreover, $\Omega_{l}$ is traceless.

The following Positive Mass Conjecture was proved first by R. Schoen and S.T. Yau [SY1, SY2, SY3], then by E. Witten [W, PT].

Theorem 1.4 (Schoen-Yau, Witten). Let $N$ be a 4-dimensional Lorentzian manifold with Lorentzian metric $\tilde{g}$ of signature $(-1,1,1,1)$, which satisfies the Einstein equations (1.1), $M \subset N$ be a spacelike asymptotically flat hypersurface of order $\tau>\frac{1}{2}$. If $M$ satisfies the dominant energy condition

$$
T_{00} \geq \sqrt{\sum_{i} T_{0 i}^{2}}, \text { and } T_{00} \geq\left|T_{\alpha \beta}\right|,
$$

then, for each end $M_{l}$, we have

$$
E_{l} \geq \sqrt{\sum_{i} p_{l i}^{2}}
$$

If $E_{l_{0}}=0$ for some $l_{0}$, then $M$ has only one end and $N$ is flat over $M$.

One key point in Witten's argument is to prove that there is a positive definite Hermitian metric on $\operatorname{Spin}(3,1)$ spinors. This fact was verified by $\mathrm{T}$. Parker and C. Taubes [PT] in terms of representation theory of spin group $S L(2, C)$, and was extended to $\operatorname{Spin}(4,1)$ spinors by the author in terms of representation theorey of spin group $H U(1,1)$. Consequently, Positive Mass 
Conjecture can be proved for spin spacelike hypersurface in 5-dimensional Lorentzian manifolds [Z1]. It should be true for all spin group $\operatorname{Spin}(n, 1)$, an issue we will address elsewhere.

Now since $N$ is 5-dimensional and $M$ is an orientable hypersurface in $N, M$ has a $\operatorname{Spin}^{c}$ structure. It means that there is a $U(1)$ line bundle $L$ on $N$ such that $S \otimes L^{\frac{1}{2}}$ is globally-defined over $M$, where $S$ is (locally) spinor bundle of $N$, which is not globally-defined on $N$ except that $N$ is spin. Denote $W=S \otimes L^{\frac{1}{2}}$. W is called the complex Witten-Dirac spinor bundle, and $L$ is called $S_{p i n}{ }^{c}$ structure. Let $A$ be a $U(1)$ connection 1-form on $L$, and denote $F_{A}^{M}$ as the curvature of $L$ restricted on $M$. The corresponding connection on $L^{\frac{1}{2}}$ is $\tilde{d}_{A}=d+\frac{1}{2} A$. Let $\nabla$ be the metric connection on $S$. Then the globally-defined connection $\nabla_{A}$ and the metric on $W$ are defined as follows: write $\phi=s_{1} \otimes \sigma_{1}, \psi=s_{2} \otimes \sigma_{2}$ locally, where $s_{1}, s_{2} \in S, \sigma_{1}^{2}, \sigma_{2}^{2} \in L$, then

$$
\begin{aligned}
\nabla_{A} \phi & =\nabla s_{1} \otimes \sigma_{1}+s_{1} \otimes \tilde{d}_{A} \sigma_{1}, \\
\langle\phi, \psi\rangle_{W} & =\left\langle s_{1}, s_{2}\right\rangle_{S} \cdot\left\langle\sigma_{1}, \sigma_{2}\right\rangle_{L} .
\end{aligned}
$$

Obviously, $\nabla_{A}$ is compatible with the metric $\langle,\rangle_{W}$. At each $p \in M$, we fix an orthonormal frame $\left\{e_{\alpha} \mid \alpha=0,1,2,3,4\right\}$ with $e_{0}$ normal to $M$ and $e_{1}, e_{2}, e_{3}, e_{4}$ tangent to $M$. (Here, and henceforth, repeated indices are summed with Latin indices running from 1 to 4 and Greek indices running from 0 to 4.) Denote $\left\{e^{\alpha} \mid \alpha=0,1,2,3,4\right\}$ as its dual frame.

Definition 1.5. The above $M$ satisfies the charged dominant energy condition if

$$
T_{00} \geq \sqrt{\sum_{i} T_{0 i}^{2}}+\sqrt{\sum_{i, j} F_{A i j}^{2}}, \text { and } T_{00} \geq\left|T_{\alpha \beta}\right|+\left|F_{A \alpha \beta}\right| .
$$

Theorem 1.6. Let $N$ be a 5-dimensional Lorentzian manifold with Lorentzian metric $\tilde{g}$ of signature $(-1,1,1,1,1)$, which satisfies the Einstein equations (1.1), $M \subset N$ be a spacelike asymptotically flat hypersurface of order $\tau>1$. Let $L$ be the Spin structure of complex Witten-Dirac spinor bundle of $M$ with $U(1)$ connection $A$, which is also asymptotically flat of order $\tau>1$. If $M$ satisfies the charged dominant energy condition (1.10), then, for each end $M_{l}$, we have

$$
E_{l} \geq\left\{\begin{array}{cc}
\sqrt{\left|Q_{l}\right|^{2}+2\left|q_{l 12} q_{l 34}+q_{l 13} q_{l 42}+q_{l 14} q_{l 23}\right|} & \text { if }\left|P_{l}\right|=0 \\
\left|P_{l}\right|+\sqrt{2^{-1}\left|Q_{l}\right|^{2}+\vec{P}_{l}^{t} \Omega_{l} \vec{P}_{l}} & \text { if }\left|P_{l}\right| \neq 0
\end{array}\right.
$$


where $\left|P_{l}\right|=\sqrt{\sum_{i} p_{l i}^{2}},\left|Q_{l}\right|=\sqrt{\sum_{i<j} q_{l i j}^{2}}$ and $\vec{P}_{l}=\left|P_{l}\right|^{-1}\left(p_{l 1}, p_{l 2}, p_{l 3}, p_{l 4}\right)^{t}$ if $\left|P_{l}\right| \neq 0$. If $E_{l_{0}}=0$ for some $l_{0}$, then $M$ has only one end and $N, L$ are flat over $M$. Moreover, $p_{l_{0} k}=0, q_{l_{0} i j}=0$.

We also prove an analogous theorem for 4-dimensional Lorentzian manifolds in the appendix. Namely,

Theorem 1.7. Let $N$ be a 4-dimensional Lorentzian manifold with Lorentzian metric $\tilde{g}$ of signature $(-1,1,1,1)$, which satisfies the Einstein equations (1.1), $M \subset N$ be a spacelike asymptotically flat hypersurface of order $\tau>\frac{1}{2}$. Let $L$ be the $\operatorname{Spin}^{c}(3,1)$ structure of $N$ with $U(1)$ connection $A$, which is also asymptotically flat of order $\tau>\frac{1}{2}$ over $M$. If $M$ satisfies the charged dominant energy condition (1.10), then, for each end $M_{l}$, we have

$$
E_{l} \geq \sqrt{\left|P_{l}\right|^{2}+\left|Q_{l}\right|^{2}+2\left|p_{l 1} q_{l 23}+p_{l 2} q_{l 31}+p_{l 3} q_{l 12}\right|}
$$

where $\left|P_{l}\right|=\sqrt{\sum_{i} p_{l i}^{2}},\left|Q_{l}\right|=\sqrt{\sum_{i<j} q_{l i j}^{2}}$. If $E_{l_{0}}=0$ for some $l_{0}$, then $M$ has only one end and $N, L$ are flat over $M$. Moreover, $p_{l_{0} k}=0, q_{l_{0} i j}=0$.

\section{Spinors.}

Let $N$ be a 5 -dimensional Lorentzian manifold, and $M$ be a spacelike hypersurface in $N$. Denote $H$ as the field of quaternions. The hyper-unitary group $H U(1,1)=\operatorname{Spin}^{0}(4,1)$ is the double covering group of connected Lorentz group $S O(4,1)$ (see [Ha], p272). A $S p i n^{c}$ structure on $N$ is a globally defined $H U(1,1) \times Z_{2} U(1)$ bundle $W$ over $M$ locally of the form $W=S \otimes L^{\frac{1}{2}}$. For any $X \in E n d(W)$, denote $X^{*}$ the adjoint of $X$ under $H U(1,1) \times{ }_{Z_{2}} U(1)$ Hermitian structure. Denote

$$
\aleph=\left\{X \in \operatorname{End}(W), X=X^{*}, \operatorname{Trace}(X)=0\right\} .
$$

There is an invariant metric on $\aleph$ defined for $X, Y \in \aleph$ by,

$$
\langle X, Y\rangle=-\frac{1}{2} \Re e(\operatorname{Trace}(X Y)) .
$$

Moreover, for any $X \in T^{*} N$ with coordinate $\left(x_{0}, x_{1}, x_{2}, x_{3}, x_{4}\right)$, we have a canonical identification of $X$ to an element in $\aleph$, i.e.,

$$
X \mapsto\left(\begin{array}{cc}
x_{0} & x \\
-\bar{x} & -x_{0}
\end{array}\right)
$$


where $x=x_{1}+x_{2} I+x_{3} J+x_{4} K$. As in [Z1] one can prove that this defines an isometry $T^{*} N \equiv \aleph$.

The spinor bundle $W$ has a $H U(1,1) \times Z_{2} U(1)$ invariant Hermitian metric defined by

$$
(\phi, \psi)=\bar{\xi}_{1} \cdot \eta_{1}-\bar{\xi}_{2} \cdot \eta_{2}
$$

for $\phi=\left(\xi_{1}, \xi_{2}\right)^{t} \in W, \psi=\left(\eta_{1}, \eta_{2}\right)^{t} \in W$. This metric is not positive definite.

The Clifford multiplication is the map $T^{*} N \otimes W \longrightarrow W$ that sends $X \otimes \phi$ to $X \phi$, where $X \phi$ means that spinor $\phi$ is mutiplied by the corresponding matrix (2.1) of covector $X$. Obviously, $X Y+Y X=-2 \tilde{g}(X, Y) \cdot I d$. The choice of a timelike covector $e^{0}$ gives another Hermitian metric on $W$ by

$$
\langle\phi, \psi\rangle=\left(e^{0} \phi, \psi\right)=\bar{\xi}_{1} \cdot \eta_{1}+\bar{\xi}_{2} \cdot \eta_{2}
$$

for $\phi=\left(\xi_{1}, \xi_{2}\right)^{t} \in W, \psi=\left(\eta_{1}, \eta_{2}\right)^{t} \in W$. This new metric is positive definite and $S p(1) \times S p(1) \times{ }_{Z_{2}} U(1)$ invariant. Furthermore, for any $X \in T_{p}^{*} N$, $x \in T_{p}^{*} M$, spinors $\phi, \psi \in W$, we have

$$
(X \phi, \psi)=(\phi, X \psi), \quad\langle x \phi, \psi\rangle=-\langle\phi, x \psi\rangle, \quad\left\langle e^{0} \phi, \psi\right\rangle=\left\langle\phi, e^{0} \psi\right\rangle .
$$

The proofs of above facts are similar to those in [Z1]. By (2.1), we get a canonical representation of the coframe

$$
\begin{aligned}
& e^{0} \mapsto\left(\begin{array}{cc}
1 & 0 \\
0 & -1
\end{array}\right), e^{1} \mapsto\left(\begin{array}{cc}
0 & 1 \\
-1 & 0
\end{array}\right), \\
& e^{2} \mapsto\left(\begin{array}{ll}
0 & I \\
I & 0
\end{array}\right), \quad e^{3} \mapsto\left(\begin{array}{cc}
0 & J \\
J & 0
\end{array}\right), \quad e^{4} \mapsto\left(\begin{array}{cc}
0 & K \\
K & 0
\end{array}\right) .
\end{aligned}
$$

Now we derive the Pauli representation. We identify $H \cong C^{2}$ as follows: For any $x_{H}=x_{1}+x_{2} I+x_{3} J+x_{4} K=\left(x_{1}+x_{2} I\right)+J\left(x_{3}-x_{4} I\right) \in H$, we identify it to $x_{C}=\left(x_{1}+x_{2} \mathbf{i}, x_{3}-x_{4} \mathbf{i}\right)^{t} \in C^{2}$. Since $I \cdot x_{H}=I\left(x_{1}+\right.$ $\left.x_{2} I\right)+J(-I)\left(x_{3}-x_{4} I\right), J \cdot x_{H}=J\left(x_{1}+x_{2} I\right)-\left(x_{3}-x_{4} I\right)$, and $K \cdot x_{H}=$ $J(-I)\left(x_{1}+x_{2} I\right)-I\left(x_{3}-x_{4} I\right)$. We can obtain the following canonical Pauli representation

$$
I \mapsto\left(\begin{array}{cc}
\mathbf{i} & 0 \\
0 & -\mathbf{i}
\end{array}\right), \quad J \mapsto\left(\begin{array}{cc}
0 & -1 \\
1 & 0
\end{array}\right), \quad K \mapsto\left(\begin{array}{cc}
0 & -\mathbf{i} \\
-\mathbf{i} & 0
\end{array}\right) .
$$

For any $x_{H}, y_{H} \in H$, we have $\Re e\left(\bar{x}_{H} y_{H}\right)=\Re e\left(\bar{x}_{C}^{t} y_{C}\right)$. This fact implies that, for any $\phi, \psi \in W, \Re e\langle\phi, \psi\rangle_{H}=\Re e\langle\phi, \psi\rangle_{C}$, where $\langle,\rangle_{H}$ is quaternions 
Hermitian metric on $W$ and $\langle,\rangle_{C}$ is the corresponding complex Hermitian metric on $W$ while $W$ is viewed as a complex rank-4 bundle.

Obviously, $W=W^{+} \oplus W^{-}$over $M$, where $W^{ \pm}=\{\phi \in W: * \phi= \pm \phi\}$ (* $\left.=-e^{1} e^{2} e^{3} e^{4}\right)$. The 'half spinor bundles' $W^{ \pm}$are orthogonal w.r.t. metrics $($,$) and \langle$,$\rangle . Moreover, since e^{0} *=* e^{0}, e^{0}$ preserves $W^{ \pm}$. Now the space of 2-forms of $M$ splits as the self-dual part $\Lambda^{+}$and the anti-self-dual part $\bigwedge^{-}, \Lambda^{ \pm}=\operatorname{span}\left\{e_{ \pm}^{I}, e_{ \pm}^{J}, e_{ \pm}^{K}\right\}$, where

$$
\begin{aligned}
& e_{ \pm}^{I}=e^{1} \wedge e^{2} \pm e^{3} \wedge e^{4}, \\
& e_{ \pm}^{J}=e^{1} \wedge e^{3} \pm e^{4} \wedge e^{2}, \\
& e_{ \pm}^{K}=e^{1} \wedge e^{4} \pm e^{2} \wedge e^{3} .
\end{aligned}
$$

Define the Clifford multiplication of 2-form on $W$ by: $\left(e^{i} \wedge e^{j}\right)=e^{i} e^{j}(i \neq j)$. A straightforward computation shows $\Lambda^{ \pm} W^{\mp}=0$. Furthurmore,

$$
\begin{aligned}
& e_{-}^{I} e^{1}=-e^{1} e_{+}^{I}, \quad e_{-}^{I} e^{2}=-e^{2} e_{+}^{I}, \quad e_{-}^{I} e^{3}=e^{3} e_{+}^{I}, \quad e_{-}^{I} e^{4}=e^{4} e_{+}^{I}, \\
& e_{-}^{J} e^{1}=-e^{1} e_{+}^{J}, \quad e_{-}^{J} e^{2}=e^{2} e_{+}^{J}, e_{-}^{J} e^{3}=-e^{3} e_{+}^{J}, e_{-}^{J} e^{4}=e^{4} e_{+}^{J}, \\
& e_{-}^{K} e^{1}=-e^{1} e_{+}^{K}, \quad e_{-}^{K} e^{2}=e^{2} e_{+}^{K}, \quad e_{-}^{K} e^{3}=e^{3} e_{+}^{K}, e_{-}^{K} e^{4}=-e^{4} e_{+}^{K},
\end{aligned}
$$

and

$$
\begin{aligned}
& e_{+}^{I} e_{+}^{J}=2 e_{+}^{K}, \quad e_{+}^{J} e_{+}^{K}=2 e_{+}^{I}, \quad e_{+}^{K} e_{+}^{I}=2 e_{+}^{J} \\
& e_{-}^{I} e_{-}^{J}=2 e_{-}^{K}, \quad e_{-}^{J} e_{-}^{K}=2 e_{-}^{I}, \quad e_{-}^{K} e_{-}^{I}=2 e_{-}^{J}
\end{aligned}
$$

\section{Hypersurface $\operatorname{Spin}^{c}$ Dirac operator.}

Let $N$ be a 5 -dimensional Lorentzian manifold, and $M$ be a spacelike hypersurface in $N$. Fix a point $p \in M$ and an orthonormal basis $\left\{e_{\alpha}\right\}$ of $T_{p} N$ with $e_{0}$ normal and $e_{1}, e_{2}, e_{3}, e_{4}$ tangent to $M$. Extend $e_{1}, e_{2}, e_{3}, e_{4}$ to an orthonormal frame in a neighbourhood of $p$ in $M$ such that $\left(\nabla_{i} e_{j}\right)_{p}=0$. Extend this to a local orthonormal frame $\left\{e_{\alpha}\right\}$ for $N$ with $\left(\widetilde{\nabla}_{0} e_{j}\right)_{p}=0$. Let $\left\{e^{\alpha}\right\}$ be the dual frame. Then $\left(\widetilde{\nabla}_{i} e^{j}\right)_{p}=-h_{i j} e^{0},\left(\widetilde{\nabla}_{i} e^{0}\right)_{p}=-h_{i j} e^{j}$, where $h_{i j}=\left\langle\widetilde{\nabla}_{i} e_{0}, e_{j}\right\rangle, 1 \leq i, j \leq 4$, are the components of the second fundamental form at $p$. The metric connection $\widetilde{\nabla}$ and $\nabla$, together with a $U(1)$ connection $A$ on $L$, induce two connections on $W$. These induced connections on $W$, which we denote by $\widetilde{\nabla}_{A}, \nabla_{A}$ respectively, are related by

$$
\widetilde{\nabla}_{A i}=\nabla_{A i}+\frac{1}{2} h_{i j} e^{0} e^{j}
$$


By definition, $\widetilde{\nabla}_{A}$ is compatible with the metric (, ), i.e.,

$$
d\left((\phi, \psi) * e_{i}\right)=\left(\left(\widetilde{\nabla}_{A i} \phi, \psi\right)+\left(\phi, \widetilde{\nabla}_{A i} \psi\right)\right) * 1 .
$$

Using (2.2) and (3.1), we can prove that $\nabla_{A}$ is also compatible with the metrics $($,$) and \langle$,$\rangle , i.e.,$

$$
\begin{aligned}
& d\left((\phi, \psi) * e_{i}\right)=\left(\left(\nabla_{A i} \phi, \psi\right)+\left(\phi, \nabla_{A i} \psi\right)\right) * 1 . \\
& d\left(\langle\phi, \psi\rangle * e_{i}\right)=\left(\left\langle\nabla_{A i} \phi, \psi\right\rangle+\left\langle\phi, \nabla_{A i} \psi\right\rangle\right) * 1 .
\end{aligned}
$$

In a local orthonormal coframe $\left\{e^{i}\right\}$ of $M, \operatorname{Spin}^{c}$ Dirac operator $D_{A}$ and the hypersurface $S p i n^{c}$ Dirac operator $\widetilde{D}_{A}$ are defined by

$$
D_{A}=e^{i} \nabla_{A i}, \quad \widetilde{D}_{A}=e^{i} \widetilde{\nabla}_{A i},
$$

respectively. Obviously, $D_{A}$ is self-adjoint with respect to the metric $\langle$,$\rangle .$ We also have the following standard Weitzenböck formula:

$$
D_{A}^{2}=\nabla_{A}^{*} \nabla_{A}+\frac{R}{4}+\frac{1}{2} F_{A}^{M},
$$

where $R$ is the scalar curvature of $M$, and $F_{A}^{M}$ is the restriction on $M$ of the curvature of $L$. From (3.1), we have

$$
\widetilde{D}_{A}=D_{A}+\frac{H}{2} e^{0}
$$

where $H=\sum h_{i i}$ is the mean curvature of $M$. Moreover,

$$
\begin{aligned}
d\left(\left\langle e^{i} \phi, \psi\right\rangle * e^{i}\right) & =\left(\left\langle D_{A} \phi, \psi\right\rangle-\left\langle\phi, D_{A} \psi\right\rangle\right) * 1 \\
& =\left(\left\langle\widetilde{D}_{A} \phi, \psi\right\rangle-\left\langle\phi, \widetilde{D}_{A} \psi\right\rangle\right) * 1 .
\end{aligned}
$$

and

$d\left(\left\langle\phi, \widetilde{\nabla}_{A i} \psi\right\rangle * e^{i}\right)=\left(\left\langle\widetilde{\nabla}_{A i} \phi, \widetilde{\nabla}_{A i} \psi\right\rangle-\left\langle\phi,\left(-\widetilde{\nabla}_{A i}+h_{i j} e^{0} e^{j}\right) \widetilde{\nabla}_{A i} \psi\right\rangle\right) * 1$.

It follows that the adjoints under the metric $\langle$,$\rangle are D_{A}^{*}=D_{A}, \widetilde{D}_{A}^{*}=\widetilde{D}_{A}$, $\widetilde{\nabla}_{A i}^{*}=-\widetilde{\nabla}_{A i}+h_{i j} e^{0} e^{j}$. With the information, we can easily derive (as in [Z1]) the following two Weitzenböck formulas,

$$
\begin{aligned}
\widetilde{D}_{A}^{2} & =\nabla_{A}^{*} \nabla_{A}+\frac{1}{4}\left(R+H^{2}\right)-\frac{1}{2} \nabla_{i} H e^{0} e^{i}+\frac{1}{2} F_{A}^{M} \\
& =\widetilde{\nabla}_{A}^{*} \widetilde{\nabla}_{A}+\frac{1}{2}\left(T_{00}+T_{0 i} e^{0} e^{i}+F_{A}^{M}\right) .
\end{aligned}
$$


The integral form of Weitzenböck formula (3.3) is

$$
\int_{M}\left|\widetilde{\nabla}_{A} \phi\right|^{2}+\langle\phi, \widetilde{R} \phi\rangle-\left|\widetilde{D}_{A} \phi\right|^{2}=\frac{1}{2} \int_{\partial M}\left\langle\phi,\left[e^{i}, e^{j}\right] \widetilde{\nabla}_{A j} \phi\right\rangle * e^{i}
$$

where $\widetilde{R}=\frac{1}{2}\left(T_{00}+T_{0 i} e^{0} e^{i}+F_{A}^{M}\right)$, and $\left[e^{i}, e^{j}\right]=e^{i} e^{j}-e^{j} e^{i}$.

Now recall that $M$ and $L$ are asymptotically flat of order $\tau>1$ with asymptotic coordinates $\left\{d x^{i}\right\}$ on the end. Orthonormalizing $\left\{d x^{i}\right\}$ yields an orthonormal coframe

$$
e^{i}=d x^{i}+\frac{1}{2} a_{i k} d x^{k}+O\left(r^{-\tau-1}\right) .
$$

Denote $e^{0}$ as $d x^{0}$. Then, on each end,

$$
\begin{aligned}
\nabla_{A j} & =\partial_{j}-\frac{1}{4} \Gamma_{k j l} d x^{k} d x^{l}+\frac{1}{2} A_{j} \mathbf{i}+O\left(r^{-2 \tau-1}\right), \\
\widetilde{D}_{A} & =d x^{j} \partial_{j}-\frac{1}{4} \Gamma_{k j l} d x^{j} d x^{k} d x^{l}+\frac{H}{2} d x^{0}+\frac{1}{2} d x^{j} A_{j} \mathbf{i}+O\left(r^{-2 \tau-1}\right),
\end{aligned}
$$

where $\Gamma_{k j l}=\frac{1}{2}\left(\partial_{j} g_{k l}+\partial_{l} g_{k j}-\partial_{k} g_{j l}\right)=O\left(r^{-\tau-1}\right)$. Therefore $\widetilde{D}_{A}$ gives the maps for the weighted Hölder spaces

$$
C_{-\tau}^{2, \alpha}(W) \stackrel{\widetilde{D}_{A}}{\longrightarrow} C_{-\tau-1}^{1, \alpha}(W) \stackrel{\widetilde{D}_{A}}{\longrightarrow} C_{-\tau-2}^{0, \alpha}(W)
$$

defined by connection $\nabla_{A}$ on $W$. Here we are using the weighted spaces defined in the papers of Bartnik [B] and Lee-Parker [LP]. For constant spinor $\phi_{0}, \partial_{j} \phi_{0}=0$, we have $\widetilde{D}_{A} \phi_{0} \in C_{-\tau-1}^{1, \alpha}(W)$, and $\widetilde{D}_{A}^{2} \phi_{0} \in C_{-\tau-2}^{0, \alpha}(W)$.

The following lemma can be easily proved in the spirit of [PT].

Lemma 3.1. Suppose $M, L$ are asymptotically flat of order $\tau>1$ and $\phi$, $\left\{\phi_{i}\right\} \in W$ are $C^{1}$ spinors which satisfy $\widetilde{\nabla}_{A} \phi=0, \widetilde{\nabla}_{A} \phi_{i}=0$ for each $i$,

(i) If $\lim _{x \rightarrow \infty} \phi(x)=0$, where the limit is taken along $M$ in one asymptotic end, then $\phi=0$.

(ii) If $\left\{\phi_{i}\right\}$ are linearly independent in some end, then they are linearly independent everywhere on $M$.

Proof. By the assumption, we have $\nabla_{A i} \phi=-\frac{1}{2} h_{i j} e^{0} e^{j} \phi$. Then

$$
\left.\left.|d| \phi\right|^{2}|=2| \Re e\left\langle\nabla_{A} \phi, \phi\right\rangle|\leq C| h|| \phi\right|^{2} .
$$

Therefore the lemma can be proved in the same way as Lemma 4.1, [Z1]. 
Lemma 3.2. If $M, L$ are asymptotically flat of order $\tau>1$ and the charged dominant energy condition (1.10) holds on $M$, then the map

$$
\widetilde{D}_{A}^{2}: C_{-\tau}^{2, \alpha}(W) \longrightarrow C_{-\tau-2}^{0, \alpha}(W)
$$

is an isomorphism.

Proof. First note that the lower order term in (3.2)

$$
\left(\frac{1}{4}\left(R+H^{2}\right)-\frac{1}{2} \nabla_{i} H e^{0} e^{i}+\frac{1}{2} F_{A}^{M}\right)
$$

lies in $C_{-\tau-2}^{0, \alpha}(W)$. Consequently, Theorem $9.2(\mathrm{~d})$ of [LP] shows that $\widetilde{D}_{A}^{2}$ is an isomorphism provided it is injective. To show injectivity, suppose that $\phi \in C_{-\tau}^{2, \alpha}(W)$ satisfies $\widetilde{D}_{A}^{2} \phi=\widetilde{\nabla}_{A}^{*} \widetilde{\nabla}_{A} \phi+\widetilde{R} \phi=0$. Integrating over the region $M_{r} \subset M$ inside radius $r$ in asymptotic coordinates, we have

$$
\int_{M_{r}}\left|\widetilde{\nabla}_{A} \phi\right|^{2}+\langle\widetilde{R} \phi, \phi\rangle=\int_{\partial M_{r}}\left\langle\phi, \widetilde{\nabla}_{A i} \phi\right\rangle * e^{i} .
$$

But

$$
\left\langle\phi, \widetilde{\nabla}_{A i} \phi\right\rangle=\left\langle\phi,\left(\nabla_{A i} \phi+\frac{1}{2} h_{i j} e^{0} . e^{j} . \phi\right)\right\rangle=O\left(r^{-2 \tau-1}\right),
$$

and $\operatorname{Vol}\left(\partial M_{r}\right)=O\left(r^{3}\right)$ by (1.2), (1.3). Hence the right hand side of the above integral vanishes in the limit as $r \rightarrow \infty$. Therefore $\widetilde{\nabla}_{A} \phi=0$ on $M$. Hence $\phi=0$ by Lemma 3.1 (i), and the proof of the lemma is complete.

Theorem 3.3. If $M, L$ are asymptotically flat of order $\tau>1$ and the charged dominant energy condition (1.10) holds on $M$, then for any constant spinor $\phi_{0}$ on ends, the following boundary value problem has a unique solution $\phi \in C^{2, \alpha}(W)$,

$$
\left\{\begin{array}{ccc}
\widetilde{D}_{A} \phi & = & 0 \\
\lim _{r \rightarrow \infty} \phi & = & \phi_{0}
\end{array}\right.
$$

Proof. Since $\widetilde{D}_{A}^{2} \phi_{0} \in C_{-\tau-2}^{0, \alpha}(W)$, Lemma 3.2 show that there is unique $\phi_{1} \in C_{-\tau}^{2, \alpha}(W)$ such that $\widetilde{D}_{A}^{2} \phi_{1}=-\widetilde{D}_{A}^{2} \phi_{0}$. Then $\phi=\phi_{1}+\phi_{0}$ satisfies $\widetilde{D}_{A}^{2} \phi=0$. Let $\psi=\widetilde{D}_{A} \phi \in C_{-\tau-1}^{1, \alpha}(W)$, then

$$
\int_{M_{r}}\left|\widetilde{\nabla}_{A} \psi\right|^{2}+\langle\widetilde{R} \psi, \psi\rangle=\int_{\partial M_{r}}\left\langle\psi, \widetilde{\nabla}_{A i} \psi\right\rangle * e^{i}=\int_{\partial M_{r}} O\left(r^{-2 \tau-3}\right) \rightarrow 0
$$

as $r \rightarrow \infty$. Therefore $\tilde{\nabla}_{A} \psi=0$ on $M$. Hence $\psi=0$ by Lemma 3.1 (i) and $\phi$ is the unique solution of (3.5). 


\section{Positive Mass Theorem.}

In this section, we will prove Positive Mass Theorem.

Proof of Theorem 1.6. Fix a constant spinor $\phi_{0} \neq 0$ on $M_{l}$ and $\phi_{0}=0$ on the other ends. Let $\phi=\phi_{0}+\phi_{1}$ be the solution of (3.5) with $\phi_{1} \in C_{-\tau}^{2, \alpha}(W)$. As in [Z1] we have

$$
\begin{aligned}
& \text { (4.1) } \int_{M}\left|\widetilde{\nabla}_{A} \phi\right|^{2}+\langle\phi, \widetilde{R} \phi\rangle \\
& =\frac{1}{2} \int_{\partial M_{\infty}}\left\langle\phi_{0},\left[d x^{i}, d x^{j}\right] \widetilde{\nabla}_{A j} \phi_{0}\right\rangle \\
& =\frac{1}{2} \int_{\partial M_{\infty}}\left\langle\phi_{0},\left[d x^{i}, d x^{j}\right] \widetilde{\nabla}_{j} \phi_{0}\right\rangle * d x^{i}+\frac{1}{4} \int_{\partial M_{\infty}}\left\langle\phi_{0},\left[d x^{i}, d x^{j}\right] A_{j} \mathbf{i} \phi_{0}\right\rangle * d x^{i} \\
& =\frac{C_{4}}{4} \sum_{l}\left(\left\langle\phi_{0}, E_{l} \phi_{0}\right\rangle+\left\langle\phi_{0}, p_{l k} d x^{0} d x^{k} \phi_{0}\right\rangle+\sum_{i<j}\left\langle\phi_{0}, d x^{i} d x^{j} q_{l i j} \mathbf{i} \phi_{0}\right\rangle\right) .
\end{aligned}
$$

We next simplify these terms algebraically. For this we temporarily drop the subscript on $\phi_{0}$, writing $\phi_{0}=\left(\phi^{+}, \phi^{-}\right) \in W^{+} \oplus W^{-}$. Similarly, we drop the subscript $l$ from $E_{l}, P_{l}, Q_{l}, \Omega_{l}, p_{l i}$ and $q_{l i j}$. When $|P| \neq 0$, we choose $\phi^{-}$so that $p_{k} d x^{0} d x^{k} \phi^{+}=-|P| \phi^{-}$. Then

$$
\begin{aligned}
\left\langle\phi_{0}, p_{k} d x^{0} d x^{k} \phi_{0}\right\rangle & =\left\langle\phi^{+}, p_{k} d x^{0} d x^{k} \phi^{-}\right\rangle+\left\langle\phi^{-}, p_{k} d x^{0} d x^{k} \phi^{+}\right\rangle \\
& =-|P|\left|\phi_{0}\right|^{2} .
\end{aligned}
$$

Denote the self-dual part of total electromagnetic momentum of end $M_{l}$ by

$$
q_{1}^{+}=2^{-1}\left(q_{12}+q_{34}\right), q_{2}^{+}=2^{-1}\left(q_{13}+q_{42}\right), q_{3}^{+}=2^{-1}\left(q_{14}+q_{23}\right),
$$

and anti-self-dual part of total electromagnetic momentum of end $M_{l}$ by

$$
q_{1}^{-}=2^{-1}\left(q_{12}-q_{34}\right), q_{2}^{-}=2^{-1}\left(q_{13}-q_{42}\right), q_{3}^{-}=2^{-1}\left(q_{14}-q_{23}\right) .
$$

Let $q^{+}=e_{+}^{I} q_{1}^{+}+e_{+}^{J} q_{2}^{+}+e_{+}^{K} q_{3}^{+}, q^{-}=e_{-}^{I} q_{1}^{-}+e_{-}^{J} q_{2}^{-}+e_{-}^{K} q_{3}^{-}$, then

$$
\begin{aligned}
\sum_{i<j}\left\langle\phi_{0}, d x^{i} d x^{j} q_{i j} \mathbf{i} \phi_{0}\right\rangle & =\left\langle\phi^{+}, q^{+} \mathbf{i} \phi^{+}\right\rangle+\left\langle\phi^{-}, q^{-} \mathbf{i} \phi^{-}\right\rangle \\
& =\left\langle\phi^{+},\left(q^{+}-|P|^{-2} p_{k} p_{j} d x^{k} q^{-} d x^{j}\right) \mathbf{i} \phi^{+}\right\rangle .
\end{aligned}
$$


Using (2.6), (2.7), (2.8), (2.9) and (2.10), we obtain

$$
\begin{aligned}
p_{k} p_{j} d x^{k} e_{-}^{I} d x^{j}= & \left(-p_{k} p_{1} d x^{k} d x^{1}-p_{k} p_{2} d x^{k} d x^{2}+p_{k} p_{3} d x^{k} d x^{3}\right. \\
& \left.+p_{k} p_{4} d x^{k} d x^{4}\right) e_{+}^{I} \\
= & \left(p_{1}^{2}+p_{2}^{2}-p_{3}^{2}-p_{4}^{2}+2 p_{1} p_{3} d x^{1} d x^{3}-2 p_{4} p_{2} d x^{4} d x^{2}\right. \\
& \left.+2 p_{1} p_{4} d x^{1} d x^{4}+2 p_{2} p_{3} d x^{2} d x^{3}\right) e_{+}^{I} \\
= & {\left[p_{1}^{2}+p_{2}^{2}-p_{3}^{2}-p_{4}^{2}+\left(p_{1} p_{3}-p_{4} p_{2}\right) e_{+}^{J}\right.} \\
& \left.+\left(p_{1} p_{4}+p_{2} p_{3}\right) e_{+}^{K}\right] e_{+}^{I} \\
= & \left(p_{1}^{2}+p_{2}^{2}-p_{3}^{2}-p_{4}^{2}\right) e_{+}^{I}+2\left(p_{1} p_{4}+p_{2} p_{3}\right) e_{+}^{J} \\
& +2\left(p_{2} p_{4}-p_{1} p_{3}\right) e_{+}^{K}
\end{aligned}
$$

Similarly, one finds that

$$
\begin{aligned}
p_{k} p_{j} d x^{k} e_{-}^{J} d x^{j}= & 2\left(p_{2} p_{3}-p_{1} p_{4}\right) e_{+}^{I}+\left(p_{1}^{2}-p_{2}^{2}+p_{3}^{2}-p_{4}^{2}\right) e_{+}^{J} \\
& +2\left(p_{1} p_{2}+p_{3} p_{4}\right) e_{+}^{K} \\
p_{k} p_{j} d x^{k} e_{-}^{K} d x^{j}= & 2\left(p_{1} p_{3}+p_{2} p_{4}\right) e_{+}^{I}+2\left(p_{3} p_{4}-p_{1} p_{2}\right) e_{+}^{J} \\
& +\left(p_{1}^{2}-p_{2}^{2}-p_{3}^{2}+p_{4}^{2}\right) e_{+}^{K}
\end{aligned}
$$

Denote

$$
\begin{aligned}
c_{1}= & q_{1}^{+}-|P|^{-2}\left(\left(p_{1}^{2}+p_{2}^{2}-p_{3}^{2}-p_{4}^{2}\right) q_{1}^{-}+2\left(p_{2} p_{3}-p_{1} p_{4}\right) q_{2}^{-}\right. \\
& \left.+2\left(p_{1} p_{3}+p_{2} p_{4}\right) q_{3}^{-}\right), \\
c_{2}= & q_{2}^{+}-|P|^{-2}\left(2\left(p_{1} p_{4}+p_{2} p_{3}\right) q_{1}^{-}+\left(p_{1}^{2}-p_{2}^{2}+p_{3}^{2}-p_{4}^{2}\right) q_{2}^{-}\right. \\
& \left.+2\left(p_{3} p_{4}-p_{1} p_{2}\right) q_{3}^{-}\right), \\
c_{3}= & q_{3}^{+}-|P|^{-2}\left(2\left(p_{2} p_{4}-p_{1} p_{3}\right) q_{1}^{-}+2\left(p_{1} p_{2}+p_{3} p_{4}\right) q_{2}^{-}\right. \\
& \left.+\left(p_{1}^{2}-p_{2}^{2}-p_{3}^{2}+p_{4}^{2}\right) q_{3}^{-}\right) .
\end{aligned}
$$


When $|P|=0$, we set

$$
\begin{aligned}
c_{1}= & q_{1}^{+}-|S|^{-2}\left(\left(s_{1}^{2}+s_{2}^{2}-s_{3}^{2}-s_{4}^{2}\right) q_{1}^{-}+2\left(s_{2} s_{3}-s_{1} s_{4}\right) q_{2}^{-}\right. \\
& \left.+2\left(s_{1} s_{3}+s_{2} s_{4}\right) q_{3}^{-}\right) \\
c_{2}= & q_{2}^{+}-|S|^{-2}\left(2\left(s_{1} s_{4}+s_{2} s_{3}\right) q_{1}^{-}+\left(s_{1}^{2}-s_{2}^{2}+s_{3}^{2}-s_{4}^{2}\right) q_{2}^{-}\right. \\
& \left.+2\left(s_{3} s_{4}-s_{1} s_{2}\right) q_{3}^{-}\right), \\
c_{3}= & q_{3}^{+}-|S|^{-2}\left(2\left(s_{2} s_{4}-s_{1} s_{3}\right) q_{1}^{-}+2\left(s_{1} s_{2}+s_{3} s_{4}\right) q_{2}^{-}\right. \\
& \left.+\left(s_{1}^{2}-s_{2}^{2}-s_{3}^{2}+s_{4}^{2}\right) q_{3}^{-}\right),
\end{aligned}
$$

where $s_{1}, s_{2}, s_{3}, s_{4}$ are arbitrary real numbers such that $|S|=\sqrt{\sum_{i} s_{i}^{2}} \neq 0$. We choose $\phi^{-}$by $s_{k} d x^{0} d x^{k} \phi^{+}=-|S| \phi^{-}$. Then we can repeat the above calculation, replacing $p_{k}$ by $s_{k}$. Therefore,

$$
\begin{aligned}
\sum_{i<j}\left\langle\phi_{0}, d x^{i} d x^{j} q_{i j} \mathbf{i} \phi_{0}\right\rangle & =\left\langle\phi^{+},\left(e_{+}^{I} c_{1}+e_{+}^{J} c_{2}+e_{+}^{K} c_{3}\right) \mathbf{i} \phi^{+}\right\rangle \\
& =2\left\langle\phi^{+},\left(I c_{1}+J c_{2}+K c_{3}\right) \mathbf{i} \phi^{+}\right\rangle .
\end{aligned}
$$

By the Pauli representation (2.4), we have

$$
\Re e \sum_{i<j}\left\langle\phi_{0}, d x^{i} d x^{j} q_{i j} \mathrm{i} \phi_{0}\right\rangle=2 \Re e\left\langle\phi^{+}, C \phi^{+}\right\rangle,
$$

where

$$
C=\left(\begin{array}{cc}
-c_{1} & -\mathbf{i} c_{2}+c_{3} \\
\mathbf{i} c_{2}+c_{3} & c_{1}
\end{array}\right)
$$

which has real eigenvalues $\lambda= \pm|C|,|C|=\sqrt{\sum_{i} c_{i}^{2}}$. Now we take $\phi^{+}$to be the eigenspinor of eigenvalue $-|C|$ with $\left|\phi^{+}\right|^{2}=\frac{1}{2}$. We obtain

$$
E-|P|-|C|=4 C_{4}^{-1} \int_{M}\left|\widetilde{\nabla}_{A} \phi\right|^{2}+\langle\phi, \widetilde{R} \phi\rangle \geq 0 \text {. }
$$

Next we compute $|C|$. Denote

$$
t_{k}= \begin{cases}|S|^{-1} s_{k} & \text { if }|P|=0 \\ |P|^{-1} p_{k} & \text { if }|P| \neq 0\end{cases}
$$


Obviously, $\sum_{k} t_{k}^{2}=1$. A straightforward computation gives

$$
\begin{aligned}
& \left(\left(t_{1}^{2}+t_{2}^{2}-t_{3}^{2}-t_{4}^{2}\right) q_{1}^{-}+2\left(t_{2} t_{3}-t_{1} t_{4}\right) q_{2}^{-}+2\left(t_{1} t_{3}+t_{2} t_{4}\right) q_{3}^{-}\right)^{2} \\
& +\left(2\left(t_{1} t_{4}+t_{2} t_{3}\right) q_{1}^{-}+\left(t_{1}^{2}-t_{2}^{2}+t_{3}^{2}-t_{4}^{2}\right) q_{2}^{-}+2\left(t_{3} t_{4}-t_{1} t_{2}\right) q_{3}^{-}\right)^{2} \\
& +\left(2\left(t_{2} t_{4}-t_{1} t_{3}\right) q_{1}^{-}+2\left(t_{1} t_{2}+t_{3} t_{4}\right) q_{2}^{-}+\left(t_{1}^{2}-t_{2}^{2}-t_{3}^{2}+t_{4}^{2}\right) q_{3}^{-}\right)^{2} \\
& =\left(q_{1}^{-}\right)^{2}+\left(q_{2}^{-}\right)^{2}+\left(q_{3}^{-}\right)^{2} .
\end{aligned}
$$

Therefore

$$
\begin{aligned}
|C|^{2} & =\left(q_{1}^{+}\right)^{2}+\left(q_{2}^{+}\right)^{2}+\left(q_{3}^{+}\right)^{2}+\left(q_{1}^{-}\right)^{2}+\left(q_{2}^{-}\right)^{2}+\left(q_{3}^{-}\right)^{2} \\
& -2\left(t_{1}^{2}+t_{2}^{2}-t_{3}^{2}-t_{4}^{2}\right) q_{1}^{+} q_{1}^{-}-4\left(t_{2} t_{3}-t_{1} t_{4}\right) q_{1}^{+} q_{2}^{-}-4\left(t_{1} t_{3}+t_{2} t_{4}\right) q_{1}^{+} q_{3}^{-} \\
& -4\left(t_{1} t_{4}+t_{2} t_{3}\right) q_{2}^{+} q_{1}^{-}-2\left(t_{1}^{2}-t_{2}^{2}+t_{3}^{2}-t_{4}^{2}\right) q_{2}^{+} q_{2}^{-}-4\left(t_{3} t_{4}-t_{1} t_{2}\right) q_{2}^{+} q_{3}^{-} \\
& -4\left(t_{2} t_{4}-t_{1} t_{3}\right) q_{3}^{+} q_{1}^{-}-4\left(t_{1} t_{2}+t_{3} t_{4}\right) q_{3}^{+} q_{2}^{-}-2\left(t_{1}^{2}-t_{2}^{2}-t_{2}^{3}+t_{4}^{2}\right) q_{3}^{+} q_{3}^{-} \\
& =\frac{1}{2}|Q|^{2}+\vec{T}^{t} \Omega \vec{T},
\end{aligned}
$$

where $\vec{T}=\left(t_{1}, t_{2}, t_{3}, t_{4}\right)^{t}$. Now we show when $|P|=0$, there is an another choice of constant spinor $\phi_{0}$ such that the third term in (4.1) has sharper value. First, by mean value inequality and (4.4),

$$
|C|^{2} \leq 2\left(\left(q_{1}^{+}\right)^{2}+\left(q_{2}^{+}\right)^{2}+\left(q_{3}^{+}\right)^{2}+\left(q_{1}^{-}\right)^{2}+\left(q_{2}^{-}\right)^{2}+\left(q_{3}^{-}\right)^{2}\right)=|Q|^{2} .
$$

On the other hand,

$$
\begin{aligned}
\Re e \sum_{i<j}\left\langle\phi_{0}, d x^{i} d x^{j} q_{i j} \mathbf{i} \phi_{0}\right\rangle & =\Re e\left\langle\phi^{+}, q^{+} \mathbf{i} \phi^{+}\right\rangle+\Re e\left\langle\phi^{-}, q^{-} \mathbf{i} \phi^{-}\right\rangle \\
& =2 \Re e\left\langle\phi^{+}, Q^{+} \phi^{+}\right\rangle-2 \Re e\left\langle\phi^{-}, Q^{-} \phi^{-}\right\rangle,
\end{aligned}
$$

where

$$
Q^{+}=\left(\begin{array}{cc}
-q_{1}^{+} & -\mathbf{i} q_{2}^{+}+q_{3}^{+} \\
\mathbf{i} q_{2}^{+}+q_{3}^{+} & q_{1}^{+}
\end{array}\right), \quad Q^{-}=\left(\begin{array}{cc}
-q_{1}^{-} & -\mathbf{i} q_{2}^{-}+q_{3}^{-} \\
\mathbf{i} q_{2}^{-}+q_{3}^{-} & q_{1}^{-}
\end{array}\right) .
$$

When $|P|=0$, we can choose $\phi^{+}, \phi^{-}$freely. So we choose $\phi^{+}$to be the eigenspinor of eigenvalue $-\left|Q^{+}\right|$of $Q^{+}$, and choose $\phi^{-}$to be the eigenspinor of eigenvalue $\left|Q^{-}\right|$of $Q^{-}$such that $\left|\phi^{+}\right|^{2}+\left|\phi^{-}\right|^{2}=1,\left|Q^{+}\right|=\sqrt{\sum_{i}\left(q_{i}^{+}\right)^{2}}$, $\left|Q^{-}\right|=\sqrt{\sum_{i}\left(q_{i}^{-}\right)^{2}}$. Then,

$$
-\Re e \sum_{i<j}\left\langle\phi_{0}, d x^{i} d x^{j} q_{i j} \mathbf{i} \phi_{0}\right\rangle=2\left|Q^{+}\right|\left|\phi^{+}\right|^{2}+2\left|Q^{-}\right|\left|\phi^{-}\right|^{2} .
$$


We choose $\phi^{-}=0$ if $\left|Q^{+}\right| \geq\left|Q^{-}\right|$, and $\phi^{+}=0$ if $\left|Q^{+}\right| \leq\left|Q^{-}\right|$. Thus

$$
\begin{aligned}
-\Re e \sum_{i<j}\left\langle\phi_{0}, d x^{i} d x^{j} q_{i j} \mathbf{i} \phi_{0}\right\rangle & =2 \max \left\{\left|Q^{+}\right|,\left|Q^{-}\right|\right\} \\
& =\sqrt{|Q|^{2}+2\left|q_{12} q_{34}+q_{13} q_{42}+q_{14} q_{23}\right|} .
\end{aligned}
$$

By (4.5), we know to get a sharper result by choose constant spinor in this way when $|P|=0$. The proof of the first part of Theorem 1.6 is complete.

Now suppose $E_{1}=0$. Then $p_{1 k}=0,1 \leq k \leq 4, c_{1 j}=0,1 \leq j \leq 3$ and $q_{1 i j}=0,1 \leq i, j \leq 4$. Take constant spinor $\left\{\psi_{1 \mu}: \mu=1,2,3,4\right\}$ which form a basis of $W$ on $M_{1}$ and $\psi_{1 \mu}=0$ on all other ends $M_{l}$, where we take $W$ as complex bundle. Let $\psi_{\mu}$ be the solutions of $\widetilde{D}_{A} \psi_{\mu}=0$ constructed from this data by Theorem 3.3. The vanishing of $E_{1}$ then implies $\widetilde{\nabla}_{A} \psi_{\mu}=0$ and $\psi_{\mu} \rightarrow 0$ uniformly on each end except $M_{1}$. But this contradicts Lemma 3.1 (i) unless $M_{1}$ is the only end of $M$. By Lemma 3.1 (ii), $\left\{\psi_{\mu}: \mu=1,2,3,4\right\}$ are linearly independent everywhere on $M$, so in a local frame $\left\{e_{i}\right\}$ of $M$,

$$
-\frac{1}{4} \widetilde{R}_{\alpha \beta i j} e^{\alpha} e^{\beta} \psi_{\mu}+\frac{1}{2} F_{A i j} \psi_{\mu}=\left(\widetilde{\nabla}_{A i} \widetilde{\nabla}_{A j}-\widetilde{\nabla}_{A j} \widetilde{\nabla}_{A i}-\widetilde{\nabla}_{A\left[e_{i}, e_{j}\right]}\right) \psi_{\mu}=0 .
$$

In terms of $(2.3),(2.4)$, we obtain

$$
\left(\begin{array}{cccc}
\widetilde{R}_{i j}^{+} \mathbf{i}-F_{A i j} & -\widetilde{R}_{i j 2}^{+}-\widetilde{R}_{i j 3}^{+} \mathbf{i} & \widetilde{R}_{i j 01}+\widetilde{R}_{i j 02} \mathbf{i} & -\widetilde{R}_{i j 03}-\widetilde{R}_{i j 04} \mathbf{i} \\
\widetilde{R}_{i j 2}^{+}-\widetilde{R}_{i j 3}^{+} \mathbf{i} & -\widetilde{R}_{i j 1}^{+} \mathbf{i}-F_{A i j} & \widetilde{R}_{i j 03}-\widetilde{R}_{i j 04} \mathbf{i} & \widetilde{R}_{i j 01}-\widetilde{R}_{i j 02} \mathbf{i} \\
\widetilde{R}_{i j 01}-\widetilde{R}_{i j 02} \mathbf{i} & -\widetilde{R}_{i j 03}+\widetilde{R}_{i j 04} \mathbf{i} & -\widetilde{R}_{i j 1}^{-} \mathbf{i}-F_{A i j} & \widetilde{R}_{i j 2}^{-}+\widetilde{R}_{i j 3}^{-} \mathbf{i} \\
\widetilde{R}_{i j 03}+\widetilde{R}_{i j 04} \mathbf{i} & \widetilde{R}_{i j 01}+\widetilde{R}_{i j 02} \mathbf{i} & -\widetilde{R}_{i j 2}^{-}+\widetilde{R}_{i j 3}^{-} \mathbf{i} & \widetilde{R}_{i j 1}^{-} \mathbf{i}-F_{A i j}
\end{array}\right) \psi_{\mu}=0
$$

where

$$
\widetilde{R}_{i j 1}^{ \pm}=\widetilde{R}_{i j 12} \pm \widetilde{R}_{i j 34}, \quad \widetilde{R}_{i j 2}^{ \pm}=\widetilde{R}_{i j 13} \pm \widetilde{R}_{i j 42}, \quad \widetilde{R}_{i j 3}^{ \pm}=\widetilde{R}_{i j 14} \pm \widetilde{R}_{i j 23}
$$

This immediately implies that, over $M, \widetilde{R}_{i j \alpha \beta}=0, F_{A i j}=0$. Therefore $T_{00}=0$ by the Einstein equations, and $\widetilde{R}_{0 i 0 j}=0, F_{A 0 i}=0$ by the charged dominant energy condition. Thus the proof of Theorem 1.6 is complete.

\section{Appendix: Analogue on 4-Lorentzian Manifolds.}

In this appendix, we assume $N$ is a 4-dimensional Lorentzian manifold with Lorentzian metric $\widetilde{g}$ of signature $(-1,1,1,1)$, which satisfies the Einstein equations (1.1), and $M$ is a spacelike hypersurface in $N$ which is asymptotically flat of order $\tau>\frac{1}{2}$. Let $L$ be a $U(1)$ line bundle which is a $\operatorname{Spin}^{c}(3,1)$ 
structure of $N$. We assume $L$ is also asymptotically flat of order $\tau>\frac{1}{2}$ over $M$. The total energy, the total linear momentum and the total electromagnetic momentum of each end of $M$ can be defined same as (1.7), (1.8), (1.9) except that we integrate over the sphere in 3-dimensional asymptotically flat ends.

Proof of Theorem 1.7. Let $V$ be (locally) $S L(2, C)$ bundle. The complex spinor bundle $W$ of $N$ is equal to $\left(\bar{V} \otimes L^{\frac{1}{2}}\right) \oplus\left(V^{*} \otimes L^{\frac{1}{2}}\right)$. Note that $L^{\frac{1}{2}}$ is globally-defined over $M$ since every orientable 3-manifold is spin. Now the Clifford multiplication can be defined as follows: For any $X \in T^{*} N$ with coordinate $\left(x_{0}, x_{1}, x_{2}, x_{3}\right)$, we identify it to the corresponding elements $X \in H o m\left(\bar{V} \otimes L^{\frac{1}{2}} \rightarrow V^{*} \otimes L^{\frac{1}{2}}\right)$ and $X^{\sigma} \in H o m\left(V^{*} \otimes L^{\frac{1}{2}} \rightarrow \bar{V} \otimes L^{\frac{1}{2}}\right)$,

$$
X \mapsto\left(\begin{array}{cc}
x_{0}-x_{1} & x_{2}+\mathbf{i} x_{3} \\
x_{2}-\mathbf{i} x_{3} & x_{0}+x_{1}
\end{array}\right), \quad X^{\sigma} \mapsto\left(\begin{array}{cc}
x_{0}+x_{1} & -x_{2}-\mathbf{i} x_{3} \\
-x_{2}+\mathbf{i} x_{3} & x_{0}-x_{1}
\end{array}\right) .
$$

Then the Clifford multiplication $T^{*} N \otimes W \longrightarrow W$ is defined by $X \otimes(\xi, \eta)^{t}=$ $\left(X \eta, X^{\sigma} \xi\right)^{t}$. We refer to [PT, Z2] for details. Now let $\phi_{0}=\left(\xi_{0}, \eta_{0}\right)^{t}$,

$$
p_{l k} d x^{0} d x^{k} \phi_{0}+\sum_{i<j} d x^{i} d x^{j} q_{l i j} \mathbf{i} \phi_{0}=\left(C_{l 1} \xi_{0}, C_{l 2} \eta_{0}\right)^{t},
$$

where

$$
\begin{aligned}
& C_{l \xi}=\left(\begin{array}{cc}
p_{l 1}-q_{l 23} & -p_{l 2}+q_{l 31}-\mathbf{i}\left(p_{l 3}-q_{l 12}\right) \\
-p_{l 2}+q_{l 31}+\mathbf{i}\left(p_{l 3}-q_{l 12}\right) & -p_{l 1}+q_{l 23}
\end{array}\right), \\
& C_{l \eta}=\left(\begin{array}{cc}
-\left(p_{l 1}+q_{l 23}\right) & p_{l 2}+q_{l 31}+\mathbf{i}\left(p_{l 3}+q_{l 12}\right) \\
p_{l 2}+q_{l 31}-\mathbf{i}\left(p_{l 3}+q_{l 12}\right) & p_{l 1}+q_{l 23}
\end{array}\right) .
\end{aligned}
$$

Note $C_{l \xi}$ has eigenvalues $\pm \lambda_{l \xi}$,

$$
\lambda_{l \xi}=\sqrt{\left(p_{l 1}-q_{l 23}\right)^{2}+\left(p_{l 2}-q_{l 31}\right)^{2}+\left(p_{l 3}-q_{l 12}\right)^{2}},
$$

and $C_{l \eta}$ has eigenvalues $\pm \lambda_{l \eta}$,

$$
\lambda_{l \eta}=\sqrt{\left(p_{l 1}+q_{l 23}\right)^{2}+\left(p_{l 2}+q_{l 31}\right)^{2}+\left(p_{l 3}+q_{l 12}\right)^{2}} .
$$

We choose spinor $\phi_{0}=\left(\xi_{0}, \eta_{0}\right)$ such that $\xi_{0}$ is the eigenspinor of eigenvalue $-\lambda_{l \xi}$ and $\eta_{0}$ is the eigenspinor of eigenvalue $-\lambda_{l \eta}$. Moreover, $\left|\xi_{0}\right|^{2}+\left|\eta_{0}\right|^{2}=1$. Then

$$
\left\langle\phi_{0}, p_{l k} d x^{0} d x^{k} \phi_{0}\right\rangle+\sum_{i<j}\left\langle\phi_{0}, d x^{i} d x^{j} q_{l i j} \mathbf{i} \phi_{0}\right\rangle=-\lambda_{l \xi}\left|\xi_{0}\right|^{2}-\lambda_{l \eta}\left|\eta_{0}\right|^{2}
$$


We choose $\eta_{0}=0$ if $\lambda_{l \xi} \geq \lambda_{l \eta}$, and $\xi_{0}=0$ if $\lambda_{l \xi} \leq \lambda_{l \eta}$. Thus, if $M$ satisfies the charged dominant energy condition, then

$$
E_{l} \geq \sqrt{\left|P_{l}\right|^{2}+\left|Q_{l}\right|^{2}+2\left|p_{l 1} q_{l 23}+p_{l 2} q_{l 31}+p_{l 3} q_{l 12}\right|} .
$$

If $E_{l_{0}}=0$ for some $l_{0}$, then $M$ has only one end, $p_{l_{0} k}=0, q_{l_{0} i j}=0$, and $\widetilde{R}_{\alpha \beta \gamma \delta}=0, F_{A \alpha \beta}=0$ over $M$. Thus the proof of Theorem 1.7 is complete. $\square$

Acknowledgements. The author would like to express his gratitude to Professor S.T. Yau for his suggestion, to Professors L.F. Tam and T. Ilmanen for their interest in this work. He would also like to thank referees for their suggestions to improve the writing of the paper. This work was partially done while the author visited Max-Planck-Institute for Mathematics in the Sciences.

\section{References.}

[ADM] S. Arnowitt, S. Deser, and C. Misner, Coordinate invariance and energy expressions in general relativity, Phys. Rev. 122 (1961), 997-1006.

[B] R. Bartnik, The mass of an asymptotically flat manifold, Comm. Pure Appl. Math. 36 (1986), 661-693.

[Ha] R. Harvey, Spinors and calibrations, Academic Press, 1989.

[HE] S. Hawking and S. Ellis, The large scale structure of space-time, Cambridge Univ. Press, 1973.

[LP] J. Lee and T. Parker, The Yamabe problem, Bull. Amer. Math. Soc. 17 (1987), 31-81.

[OW] J. Overduin and P. Wesson, Kaluza-Klein Gravity, gr-qc/9805018.

[PT] T. Parker and C. Taubes, On Witten's proof of the positive energy theorem, Comm. Math. Phys. 84 (1982), 223-238.

[SY1] R. Schoen and S.T. Yau, On the proof of the positive mass conjecture in general relativity, Comm. Math. Phys. 65 (1979), 45-76.

[SY2] R. Schoen and S.T. Yau, The energy and the linear momentum of spacetimes in general relativity, Comm. Math. Phys. 79 (1981), 47-51.

[SY3] R. Schoen and S.T. Yau, Proof of the positive mass theorem, II, Comm. Math. Phys. 79(1981), 231-260. 
[W] E. Witten, A new proof of the positive energy theorem, Comm. Math. Phys. 80 (1981), 381-402.

[Z1] X. Zhang, Positive mass conjecture for 5-dimensional Lorentzian manifolds, J. Math Phys. 40 (1999), 3540-3552.

[Z2] X. Zhang, Positive mass theorem for modified energy condition, preprint.

Institute of Mathematics, Chinese Academy of Sciences

BeiJing 100080, P.R. China

E-mail addresses: xzhang@math08.math.ac.cn

ReCeived June 1, 1998. 